Considering the bone status, patients with Hcy showed a significantly lower TBS $(p=0.03)$; the average values of BMD on the lumbar spine $(p=0.79)$ and femoral neck $(p=0.13)$ were found lower compared to, but without any statistical significance. Furthermore, no significant differences were observed in bone turnover markers according to Hcy levels.

Conclusion: The study demonstrates a relationship between higher levels of Hcy and lower TBS values within SSc patients, particularly in those with most severe microvascular damage al NVC ("Late" SSc pattern). Therefore it is concluded that higher serum levels of Hcy associate to both bone microarchitectural and microvascular damage in SSc.

REFERENCES:

[1] Cutolo M et al Expert Rev Clin Immunol 2019; 15: 753-64

[2] Behera J et al. J Cell Physiol 2017;232(10):2704-2709

[3] Yan-lie Zhang et al. Rheumatol 2018; 28(4):681-689

Disclosure of Interests: None declared

DOI: 10.1136/annrheumdis-2021-eular.2520

\begin{tabular}{|l|l}
\hline AB0439 & CAPILLAROSCOPIC PATTERNS IN PATIENTS \\
WITH SYSTEMIC SCLEROSIS-POLYMYOSITIS/ \\
DERMATOMYOSITIS (SSC-PM/DM) OVERLAP SYNDROME
\end{tabular}

O. Desinova ${ }^{1}$, M. Starovoytova ${ }^{1}$, L. P. Ananyeva ${ }^{1}{ }^{1}$ VA Nasonova Research Institute of Rheumatology, Laboratory of Microcirculation and Inflammation, Moscow, Russian Federation

Background: Impaired microcirculation is one of the leading factors in local and general pathogenesis of SSc. Widefield nail-fold video-capillaroscopy (NFVC) stands as the most informative and at the same time simple method used for evaluation of capillary circulation.

Objectives: To identify characteristic and specific for SSc- PM/DM capillaroscopic features.

Methods: Both hand II - V fingers of 68 pts with SSc-PM/DM were subjected to widefield NFC, evaluated using a binocular 20x magnification Olympus microscope and analyzed in view of specific skin lesions discriminating diffuse and limited SSc forms.

Results: SSc-specific dilatations of capillary loops were the most common for SSc-PM/DM and were found in all pts; $50 \%$ of them had signs of active scleroderma pattern, such as capillary loss or "avascular areas" (50\%) and hemorrhages (51.5\%), associated with generalized microvascular spasm in early disease and capillary sclerosis in advanced disease. The morphological capillary abnormalities such as varying degrees of capillary loops tortuosity/vascular inhomogeneity were present in $63 \%$ of examined nailfolds, branching bushy behavior of capillary loops and mega-capillaries predominated; architectural disorientation/disarrangement of capillary loops with formation of subcutaneous plexus was seen in more than $50 \%$ of them. Capillaroscopic changes consistent with active scleroderma pattern were present in $54 \%$ and were associated with lab signs of inflammatory muscle syndrome and immunological disorders: giant capillaries $(p<0.02)$, disorientation of capillary loops $(p<0.02)$ and ramified/ bushy capillaries $(p<0.04)$ were significantly more frequent in patients with severe muscle syndrome, increased CPK, ANF -positivity and hemorrhages $(p<0.03)$.

Conclusion: Thus, widefield NFVC revealed a "mixed" nature of capillaroscopic changes, combining features specific for SSc (capillary dilation, avascular areas, hemorrhages) and for PM/DM (bushy and giant capillaries, disorientation of capillary loops of the nailfold with formation of subcutaneous plexuses.)

Disclosure of Interests: None declared

DOI: 10.1136/annrheumdis-2021-eular.2540

\section{AB0440 CLINICAL AND IMMUNOLOGICAL FEATURES OF THE SYSTEMIC SCLEROSIS-OVERLAP SYNDROMES}

O. Desinova ${ }^{1}$, M. Starovoytova ${ }^{1}$, L. P. Ananyeva1, O. Koneva1, L. Garzanova ${ }^{1}$, R. Shayakhmetova', O. Ovsyannikova ${ }^{1}$. ${ }^{1}$ VA Nasonova Research Institute of Rheumatology, Laboratory of Microcirculation and Inflammation, Moscow, Russian Federation

Background: Systemic Sclerosis (SSc) overlap syndromes (SSc with polymyositis / dermatomyositis (PM/DM), rheumatoid arthritis (RA), etc.) still remain a group of very heterogenous and not very well studied clinical variants of SSc that are characterized by certain clinical and immunological features.

Objectives: Identify clinical and immunological features of the SSc-overlap syndromes

Methods: 80 pts with SSc-PM/DM and 35 pts with SSc-RA undergoing standard clinical examination and laboratory immunological evaluation.

Results: ANA Hep2 was positive in 98\% of SSc-PM/DM pts; a-Scl-70 was in 34\%, a - PM-Scl and RF were in 20\%. ACA (6\%), a-RNP (9\%), and a - Jo-1 (5\%) were significantly less common. Correlation analysis showed significant prevalence of conduction abnormalities in pts with a-Scl-70- $(p<0.03)$; PM-Scl was rarely associated with cardiac arrhythmia $(p<0.02)$ and pericarditis $(p<0.03)$, but there was an association between ACA and presence of digital ischemia $(p<0.04)$. Three pts with limited skin had Scl-70 and PM-Scl antibodies, two of them manifested clinical features of DM. A-Jo-1 was found in 3 pts with a longstanding disease $(14,10$ and 7 years), and one of these pts was also positive for a-Scl-70. All pts had limited skin and two had interstitial lung disease with FVC values of $79 \%$ and $74.8 \%$.

ANA Hep2 was positive in $96 \%$ of SSc-RA pts; a-Scl- 70 - in $28 \%$, and a-RNP - in $30 \%$. RF-positivity was in $72 \%$ of pts, and Anti-CCP - in $27 \%$. Simultaneous AntiCCP and a-Scl-70 was found in one case, and Anti-CCP - anti-RNP - in another both were associated with low RF titers. All pts had early joint involvement which became prevailing in subsequent years, and onset of the disease between 30 and 36 years. There was a correlation between laboratory signs of inflammatory activity and immunological disorders: ESR and a-Scl-70 ( $p<0.03)$. Anti-CCP and a-Scl-70 co-positivity was a significantly less frequent phenomenon $(p<0.04)$ There was a remarkable $28 \%$ proportion of a-Scl-70 cases in SSc-RA with limited cutaneous which is usually characterized by ACA-positivity.

Conclusion: SSc-PM/DM and SSc-RA appear to be an active disease from the immunological point of view, confirming therefore an important role of immune alterations in disease progression. Laboratory findings display specific pathogenetic features of SSc-overlap syndromes; laboratory abnormalities can be used to measure the activity and specify characteristics of the pathological process.

Disclosure of Interests: None declared

DOI: 10.1136/annrheumdis-2021-eular.2550

\section{$\mathrm{AB} 0441$ \\ NAILFOLDCAPILLAROSCOPY AND CANDIDATE BIOMARKER LEVELS IN SYSTEMIC SCLEROSIS- ASSOCIATED PULMONARY HYPERTENSION; PROFILING OF NON-INVASIVE MARKERS, A COHORT STUDY}

J. Lemmers $^{1}$, C. H. M. Van den Ende ${ }^{1}$, R. Smeets ${ }^{2}$, B. Kersten ${ }^{1}$, A. Van Caam ${ }^{3}$, S. Van Leuven ${ }^{1}$, J. Van Haren-Willems ${ }^{4}$, A. Van Dijk ${ }^{5}$, M. Vonk ${ }^{1} .{ }^{1}$ Radboud University Medical Center, Rheumatology, Nijmegen, Netherlands; ${ }^{2}$ Radboud University Medical Center, Laboratory for Clinical Chemisty, Nijmegen, Netherlands; ${ }^{3}$ Radboud University Medical Center, Laboratory of Experimental Rheumatology, Nijmegen, Netherlands; ${ }^{4}$ Radboud University Medical Center, Pulmonology, Nijmegen, Netherlands; ${ }^{5}$ Radboud University Medical Center, Cardiology, Nijmegen, Netherlands

Background: Systemic sclerosis (SSc) is characterized by inflammation, vascu lopathy and progressive fibrosis. Pulmonary Hypertension $(\mathrm{PH})$ is one of the leading causes of death in SSc (1). Currently, most patients with SSc are screened for the presence of $\mathrm{PH}$, and focus lies on early detection and early treatment Recent literature describes potential for both nailfoldcapillaroscopy (NCM) and several biomarkers to serve as non-invasive tools to identify SSc patients at risk for developing $\mathrm{PH}$ (2). Ideally this could attribute to further improvement of risk stratification in SSc and $\mathrm{PH}$ screenings algorithms.

Objectives: To explore NCM characteristics and plasma levels of selected candidate biomarkers in a cross-sectional cohort of SSc patients with and without different forms of $\mathrm{PH}$.

Methods: From 02-2018 until 02-2019 we included 40 consecutive SSc patients with associated $\mathrm{PH}$ (30 (75\%) were female, 32 (80\%) LcSSc, median age 72 years (IQR 69-77), median SSc duration 10.7 years (IQR 4.3-17.8), median PH duration 3.9 years (IQR 1.5-7.06)) and 40 without $\mathrm{PH}(28(70 \%)$ were female, $26(65 \%)$ had a LcSSc, median age 59 years (IQR 51-71), median SSc duration 6 years (IQR 3.1-8.6). In each group NCM characteristics (both quantitative and qualitative) and plasma levels of IL4, IL6, IL8, IL13, PDGFAA, PDGFAB-BB, 6Ckine, sTRAIL, MMP1, MMP7, sICAM1, sVCAM, CCL19/MIP3b, Endostatin, sVEGFR1, sVEGFR2, sVEGFR3, CXCL4, Endothelin1, FGF1, FGF2, VEGF-A, VEGF-C, and VEGF-D using Luminex kits, and vascular auto-antibodies AT1R and ETAR using ELISA (Celltrend $\mathrm{GmbH}$, Luckenwalde, Germany) were determined. NCM characteristics were compared using t-tests, biomarker levels were compared by using Mann-Whitney $U$ tests.

Results: We observed no differences in mean capillary density, number of abnormally shaped capillaries, number of fingers with density $<3$ capillaries $/ \mathrm{mm}$ and in overall NCM pattern between patients with and without $\mathrm{PH}$. Plasma levels showing significant differences between the two groups are presented in table 1. Conclusion: We found significant differences in several of the selected biomarkers in SSc patients with and without $\mathrm{PH}$, but not in NCM characteristics between the groups. However, we did observe a tendency toward more morphologic abnormalities and an overall late pattern in the SSc-PH group. Future longitudinal research should explore the added value of these NCM parameters and biomarkers in personalized risk stratification for the development of $\mathrm{PH}$.

\section{REFERENCES:}

[1] Elhai M, Meune C, Boubaya M, Avouac J, Hachulla E, Balbir-Gurman A, et al Mapping and predicting mortality from systemic sclerosis. Ann Rheum Dis. 2017;76(11):1897-905.

[2] Guillén-Del-Castillo A, Simeón-Aznar CP, Callejas-Moraga EL, Tolosa-Vilella C, Alonso-Vila S, Fonollosa-Pla V, et al. Quantitative videocapillaroscopy correlates with functional respiratory parameters: a clue for vasculopathy as a pathogenic mechanism for lung injury in systemic sclerosis. Arthritis Res Ther. 2018;20(1):281. 
Table 1.

\begin{tabular}{|c|c|c|c|}
\hline $\mathrm{NCM}$ & $\begin{array}{l}\mathrm{SSc} P H \\
(\mathrm{n}=40)\end{array}$ & $\begin{array}{c}\text { SSc noPH } \\
(n=38,2 \text { missing })\end{array}$ & $P$ value \\
\hline Mean capillary density/mm $( \pm \mathrm{SD})$ & $5.0( \pm 1.4)$ & $5.4( \pm 1.6)$ & 0.39 \\
\hline $\begin{array}{l}\text { Mean number of fingers with } \\
\text { density }<3( \pm S D)\end{array}$ & $2.3( \pm 1.9)$ & $1.7( \pm 2.1)$ & 0.20 \\
\hline $\begin{array}{l}\text { Mean abnormally shaped capillar- } \\
\text { ies/ } \mathrm{mm}( \pm \mathrm{SD})\end{array}$ & $1.2( \pm 0.46)$ & $0.99( \pm 0.53)$ & 0.07 \\
\hline NCM pattern $(\mathrm{n})$ & & & 0.103 \\
\hline Normal/aspecific & 1 & 3 & \\
\hline early & 0 & 3 & \\
\hline active & 4 & 7 & \\
\hline late & 34 & 25 & \\
\hline Not interpretable & 1 & 2 & \\
\hline Biomarkers* & $\begin{array}{c}\text { SSc } \mathrm{PH} \\
(\mathrm{n}=33,7 \text { missing) } \\
\text { IQR) }\end{array}$ & $\begin{array}{c}\text { SSc noPH } \\
(n=40) \\
\text { (median, IQR) }\end{array}$ & $P$ value \\
\hline IL6 pg/ml & $4.8(1.7-8.2)$ & $2.5(1.3-5.6)$ & 0.047 \\
\hline PDGF-AB-BB pg/ml & 29535 (24777-33458) & 32221 (29409-37549) & 0.0034 \\
\hline sTRAIL pg/ml & $36.6(20,4-50,6)$ & $60.8(40.7-82.4)$ & $<0.0001$ \\
\hline sVCAM1 ng/ml & $311(254-374)$, & $265(228-323)$ & 0.0236 \\
\hline Endostatin ng/ml & $53.8(41.9-72.6)$ & $39(28.6-46)$ & $<0.0001$ \\
\hline sVEGFR2 pg/ml & 10388 (7276- 13323) & $13736(10282-16554)$ & 0.0006 \\
\hline $\mathrm{CXCL} 4 \mathrm{ng} / \mathrm{ml}$ & 3347 (2546-4708) & $5309(4089-6322)$ & $<0.0001$ \\
\hline VEGF-A pg/ml & $672(248-955)$ & $346(175-703)$ & 0.0424 \\
\hline VEGF-D pg/ml & $671(421-1012)$ & $515(262-734)$ & 0.0296 \\
\hline
\end{tabular}

* only significant values with a $p<0.05$ are shown

Disclosure of Interests: Jacqueline Lemmers: None declared, C.H.M. van den Ende: None declared, R. Smeets: None declared, Brigit Kersten: None declared, Arjan van Caam: None declared, Sander van Leuven: None declared, Jolanda van Haren-Willems: None declared, Arie van Dijk Speakers bureau: Actelion(Janssen), Consultant of: Actelion(Janssen), Grant/research support from: Unrestricted educational Grant for PhD student from Actelion (Janssen), Madelon Vonk Speakers bureau: Actelion(Janssen), Boehringer Ingelheim, Roche, Consultant of: Advisory Board from Actelion(Janssen) and Boehringer Ingelheim, Grant/research support from: Unrestricted Educational Grant and research support from Actelion(Janssen), research support from Ferrer DOI: 10.1136/annrheumdis-2021-eular.2722

\section{AB0442 $\quad$ CLINICAL FEATURES OF POLYMYOSITIS AND DERMATOMYOSITIS PATIENTS WITH SEVERE DYSPHAGIA}

Y. Hayashi ${ }^{1}$, K. Izumi ${ }^{1}$, S. Hama ${ }^{1}$, M. Higashida-Konishi ${ }^{1}$, M. Ushikubo ${ }^{1}$ H. Oshima ${ }^{1}$, Y. Okano ${ }^{1} .{ }^{1}$ National Hospital Organization Tokyo Medical Center, Rheumatology, Tokyo, Japan

Background: Polymyositis (PM) and dermatomyositis (DM) are autoimmune inflammatory diseases characterized by proximal myositis. Dysphagia has been reported to develop in 35 to $62 \%$ of PM/DM patients and known as poor prognosis factor.

Objectives: The purpose of this study is to determine the clinical characteristics of PM/DM patients who present with deglutition disorder.

Methods: Consecutive patients with PM/DM who visited National Hospital Organization Tokyo Medical Center between April 2010 and January 2021 are included in this study. We compared clinical features between the patients with and without dysphagia. The diagnosis of dysphagia was based on videofluorography swallow study, and dysphagia requiring gastrostomy was defined as severe dysphagia. The clinical characteristics compared in this study were following: age of onset, levels of serum creatine kinase (CK) and lactate dehydrogenase(LDH), sense of dysphagia, manual muscle test (MMT) score, and complication of malignancy or interstitial pneumonia.

Results: A total of 73 patients with PM/DM were identified. Among them, 12 patients were diagnosed with dysphagia, and 5 patients developed severe dysphagia. Patients with dysphagia had the following characteristics compared to patients without dysphagia: higher levels of serum LDH $(833.7 \pm 500.1 \mathrm{U} / \mathrm{L}$ vs $471.9 \pm 321.0 \mathrm{U} / \mathrm{L}, \mathrm{p}=0.0088)$, higher levels of serum CK at initial examination $(6070.3 \pm 7184.8 \mathrm{IU} / \mathrm{L}$ vs $1534.7 \pm 2978.8 \mathrm{IU} / \mathrm{L}, \mathrm{p}=0.0086)$ and more frequent sense of dysphagia $(90.9 \%$ vs $10.6 \%, p<0.0001)$, lower MMT score(3.18 \pm 1.07 vs $4.31 \pm 0.75, p=0.0017)$. In addition to those, patients with severe dysphagia presented older age of onset (mean age $69.4 \pm 12.0$ vs $51.7 \pm 14.8, p=0.014$ ), more frequent complication of malignancy $(80.0 \%$ vs $14.8 \%, p=0.0048)$ and less frequent complication of interstitial pneumonia ( $0.0 \%$ vs $55.5 \%, p=0.023)$. Conclusion: These results indicate that dysphagia develops frequently in $\mathrm{PM} /$ DM patients with higher levels of serum LDH or CK, sense of dysphagia and low MMT score. Among them, patients with elderly onset or malignancy are at risk for sever dysphagia, and should be treated carefully.

Disclosure of Interests: None declared.

DOI: 10.1136/annrheumdis-2021-eular.2757

\section{$\mathrm{AB} 0443$ \\ BIOMARKER SUCH AS IL-17, IL-21 AND TIMP-1, IS USEFUL FOR PREDICTING THE PATHOPHYSIOLOGY OF CONNECTIVE TISSUE DISEASE-ASSOCIATED PULMONARY HYPERTENSION}

S. Nakashima ${ }^{1}$, T. Kameda ${ }^{1}$, H. Shimada ${ }^{1}$, M. Mahmoud Fahmy Mansour ${ }^{1}$, R. Wakiya ${ }^{1}$, M. Kato ${ }^{1}$, Y. Ushio ${ }^{1}$, K. Sugihara ${ }^{1}$, H. Dobashi ${ }^{1}{ }^{1}$ Kagawa University, Division of hematology, Rheumatology and Respiratory Medicine, Department of Internal medicine, Kagawa, Japan

Background: Connective tissue disease-associated pulmonary hypertension (CTD-PH) is constructed by a variety of pathologies, including cardiac, pulmonary, and vascular involvement, as well as immune abnormalities. Because of its various constructs, it is difficult for many respiratory physicians, cardiologists, and rheumatologists to determine a treatment strategy for CTD-PH. In addition, CTD-PH has different pathologies from $\mathrm{PAH}$, and there are cases in which immunosuppressive therapy is effective. These suggests that the two PAHs may have different pathogenesis, including inflammation in the pulmonary artery. However, there are not enough biomarkers to distinguish pathologies. On the other hand, it has been reported that various cytokines such as TIMP-1, Interleukin (IL)-6, IL-17, and IL-21 are involved in the pathogenesis of CTD-PH or vasculitis. $(1,2)$ However, the relationship between these cytokine expression and the pathogenesis or treatment of CTD-PH has not been fully clarified.

Objectives: To clarify the relationship between cytokine profile and clinical features, change in cytokines and hemodynamics by treatment, association with the effectiveness of immunosuppressive therapy.

Methods: Patients suspected PH was included. At the time of cardiac catheterization( $\mathrm{RHC})$, sera in pulmonary pre and post-capillary were collected and TIMP-1, MCP-1, IL-17 and IL-21, IL-12p70 and IL-6 were analyzed by ELISA(ABCAM UK, Ella simple plex USA). The following clinical data were collected: age, gender, underlying disease, complication of interstitial lung disease, treatment (immunosuppressant and pulmonary vasodilator), hemodynamics. Furthermore, we investigated the relationship between cytokines and clinical data.

Results: 15 cases of CTD-PH, 13 cases of non-CTD-PH, and 6 cases of non-PH were analyzed. (SSc 12 cases, MCTD 7cases, SLE 2 cases, and others 13 cases) 28 cases were diagnosed with $\mathrm{PH}$ by $\mathrm{RHC}$. There was a positive correlation between IL- 6 and mean pulmonary arterial pressure in all $\mathrm{PH}$ case. In addition, MCP-1, IL-6, and TIMP-1 tend to be high in SSc-PH cases. On the other hand in Non-SSc-PH, IL-12p70 and IL-17 were high. In cases who pulmonary vascular hemodynamics improved by treatment, IL-17, IL-21, and TIMP-1 decreased.

Conclusion: Biomarker profiles in pulmonary capillaries may differ depending on the disease. Furthermore, it suggested that IL-17, IL-21 and TIMP-1 may be biomarkers of therapeutic effect.

\section{REFERENCES:}

[1] Hashimoto-Kataoka T. et al. Proc Natl Acad Sci U S A. 2015 May 19;112(20):E2677-86.

[2] Jun Ishizaki et al. Arthritis Res Ther. 2017 Sep 29;19(1):218

Disclosure of Interests: None declared.

DOI: 10.1136/annrheumdis-2021-eular.2765

\section{AB0444 CLINICAL-IMMUNOLOGICAL CHARACTERISTICS OF PATIENTS WITH INFLAMMATORY MYOPATHIES}

A. Khelkovskaia-Sergeeva ${ }^{1}$, O. Desinova ${ }^{1}$, M. Starovoytova ${ }^{1}$, L. P. Ananyeva ${ }^{1}$, M. Cherkasova ${ }^{2}, \mathrm{R}$. Shayakhmetova ${ }^{1}{ }^{1}$ VA Nasonova Research Institute of Rheumatology, Laboratory of Microcirculation and Inflammation, Moscow, Russian Federation; ${ }^{2}$ VA Nasonova Research Institute of Rheumatology, Laboratory of Immunology and Molecular Biology, Moscow, Russian Federation

Background: Inflammatory disorders of the skeletal muscle include polymyositis (PM), dermatomyositis (DM), amyopathic dermatomyositis ADM, (immune mediated) necrotizing myopathy (NM), overlap syndrome with myositis (overlap myositis, OM) and including anti-synthetase syndrome (ASS).

The detection of myositis-specific antibodies, the clinical effect of which remains to be determined, may be of great importance for diagnosis.

Objectives: To study clinical-immunological characteristics of patients with inflammatory myopathies.

Methods: 57 pts were enrolled in this study: 28 (47\%) pts were diagnosed with DM, 14 (23\%) pts - OM, 5(8\%) pts - PM, 5 (8\%) pts-NM, 4 (7\%) pts-ADM, 1 (2\%)- ACC. Duration of disease in average 11,29 (2-48) month. Standard clinical examination and laboratory immunological evaluation including myositis-specific autoantibodies were performed.

Results: There were 3 age groups: $18-39$ years old - 16 (28\%) pts, 40-49 yo $-28(49 \%)$ pts and > 60 yo $-13(33 \%)$ pts. Symptoms of myositis were muscle weakness $57(100 \%)$ pts, dysphagia $29(51 \%)$ pts, arthritis $-12(21 \%)$ pts, Raynaud's phenomenon 21 (37\%) pts, skin lesions- 37 (65\%) pts (Gottron signs- 7 $(12 \%)$ pts, digital ulcers -11 (19) pts, panniculitis $-6(11 \%)$ pts, hand of mechanic $16(28 \%)$ pts). MMT 8 was 55,57 (SD 18,49) $(20-80)$ score. Clinical features of myositis were myocarditis $8(14 \%)$ pts and arrhythmia $9(16 \%)$ pts, interstitial lung 